\title{
Hyperbranched Poly (Ester)S from renewable Bio Monomers by Design: Nontoxic Platforms for the delivery of therapeutic agents
}

\author{
Bob A Howell ${ }^{1 *}$, Tracy Zhang ${ }^{1,2}$ and Patrick R Smith \\ ${ }^{1}$ Department of Chemistry and Biochemistry, Central Michigan University, USA \\ ${ }^{2}$ Michigan State University-St. Andrews, USA
}

*Corresponding author: Bob A Howell, Science of Advanced Materials, Center for Applications in Polymer Science, Department of Chemistry and Biochemistry, Central Michigan University, Mt. Pleasant, MI 48859-0001, USA.

Received Date: September 19, 2019

Published Date: October 11, 2019

\section{Abstract}

Hyperbranched poly(ester)s from adipic or succinic acid and glycerol generated under conditions which permit high monomer conversion without gelation and assure the presence of hydroxyl endgroups may serve as effective platforms for the delivery of therapeutic agents. Active agents may be attached by esterification at the end groups. Smooth, sustained release of the therapeutic agent may be achieved hydrolytically.

\section{Introduction}

Hyperbranched poly(ester)s produced from nontoxic, renewable biosources offer great potential for the delivery of therapeutic agents. In general, hyperbranched poly(ester)s have been produced from multifunctional monomers without regard for gelation (coatings industry) or with reaction conditions determined empirically to avoid gelation [1-6]. This approach does not permit reproducibility of polymer molecular weight, dispersity, structure or level of end group functionality. It lacks economy in time and reagents, is not amenable to scale-up, and is intellectually unsatisfying. A better method is needed for the generation of delivery vehicles and has been developed [7-9].

For the polymerization of monomers containing functional groups of unequal reactivity, e.g. glycerol (the primary hydroxyl groups of glycerol are approximately 1.4 times as reactive in esterification as is the secondary hydroxyl group) traditional gelation theory is inadequate for selecting appropriate monomer ratios (ratios of reactive functional groups) to assure a well-defined, non-gelling structure at high monomer conversion [7]. However, the Mascosko-Miller conditional probability model is suitable for this purpose [10-12]. This approach has been demonstrated for the synthesis of hyperbranched poly(ester)s from a range of bio monomers [7-9]. Using Macosko-Miller theory an appropriate ratio of monomers may be selected to avoid gelation at high conversion and to ensure the presence of desired endgroups for the attachment of therapeutic agents [8]. This is illustrated below for the generation of a salicylate prodrug suitable for controlled release of the active component.

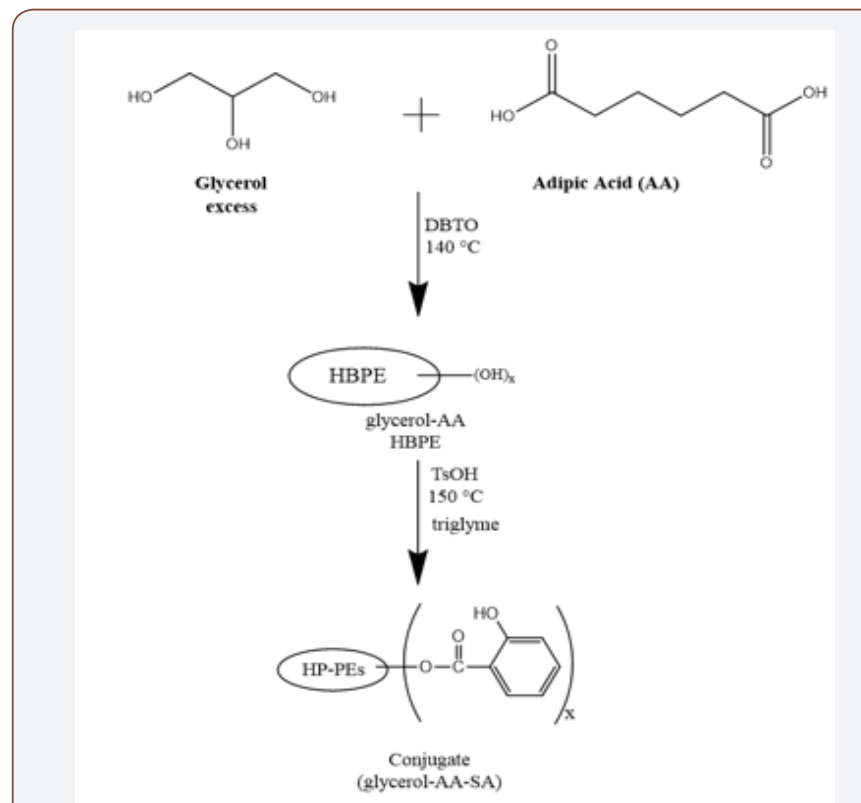

Figure 1: Preparation of Glycerol/Adipic Acid. Hyperbranched Poly(ester)/Salicylic Acid Conjugate. 
A Naproxen conjugate may be prepared in an analogous manner. A glycerol/succinic acid hyperbranched poly(ester) may also be used for the preparation of similar prodrugs. Salicylic acid is a common therapeutic used for the treatment of acne and inflammation [13-15]. Controlled release of the active agent from the drug/polymer conjugate would enhance the duration of effectiveness while minimizing skin irritation. Release of the active agent occurs smoothly from either conjugate under either chemical or enzymatic (rat liver microsomes) catalysis.

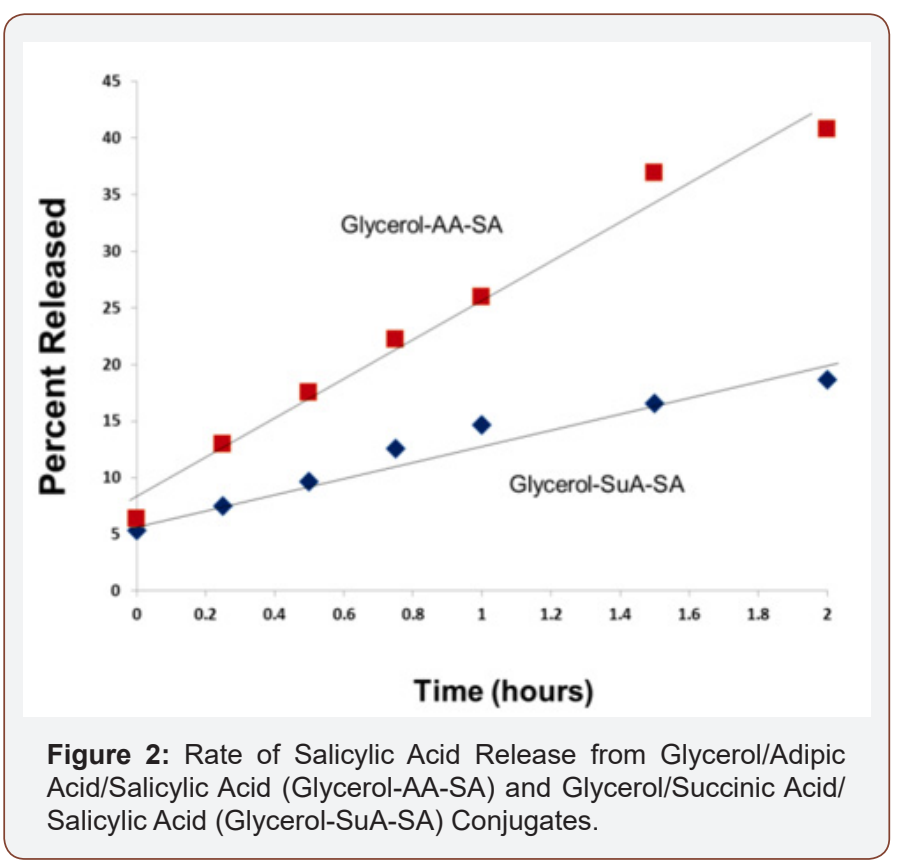

In summary, using Macosko-Miller statistics, conditions for the polymerization of multifunctional bio monomers may be predetermined to permit high monomer conversion without gelation and to assure the presence desired end group functionality. Hyperbranched glycerol poly(ester)s of both adipic and succinic acid containing hydroxyl endgroups have been prepared and converted to conjugates via esterification with active therapeutic agents. Hydrolytic release of the active agent occurs smoothly under enzymatic catalysis.

\section{References}

1. JF Stumbe, B Bruchmann (2004) Hyperbranched Polyesters Based on Adipic Acid and Glycerol. Macromolecular Rapid Communications 25(9): 921-924.

2. VT Wyatt (2012) Lewis Acid-catalyzed Synthesis of Hyperbranched Polymers Based on Glycerol and Diacids in Toluene. Journal of the American Oil Chemists' Society 89(2): 313-319.

3. HR Kricheldorf, G Behnken (2018) Biodegradable Hyperbranched Aliphatic Polyesters Derived from Pentaerythritol. Macromolecules 41(15): 5651-5657.

4. H Cao, Y Zheng, J Zhou, W Wang, A Pandit (2011) A Novel Hyperbranched Polyester Made from Aconitic Acid $\left(B_{3}\right)$ and Di (ethylene glycol) $\left(A_{2}\right)$. Polymer International 60(4): 630-634.

5. Y Li, WD Cook, C Moorhoff, WC Huang, QZ Chen (2013) Synthesis, Characterization and Properties of Biocompatible Poly (glycerol sebacate) Pre-polymer and Gel. Polymer International 62(4), 534-547.

6. H Saeed, Y Eltahir, Y Xia, Y Wang (2014) Synthesis and Characterization of A2+B4 Hyperbranched Polyesters with Hydroxyl End Groups. Advanced Materials Research 937: 80-85.

7. T Zhang, BA Howell, PB Smith (2017) Rational Synthesis of Hyperbranched Poly(ester)s. Ind Eng Chem Res 56: 1661-1670.

8. T Zhang, PB Smith, BA Howell (2019) Controlled Synthesis of Hyperbranched Poly(ester)s from Bio renewable Monomers for the Delivery of Therapeutic Agents. Glob J Eng Sci 3(1).

9. T Zhang, BA Howell, A Dumitrascu, SJ Martin, PB Smith (2014) Synthesis and Characterization of Glycerol-Adipic Acid Hyperbranched Poly(ester) s. Polymer 55(20): 5065-5072.

10. CW Macosko, DR Miller (1976) A New Derivation of Average Molecular Weights of Nonlinear Polymers. Macromolecules 9(2): 199-206.

11. DR Miller, CW Macosko (1978) Average Property Relations for Nonlinear Polymerization with Unequal Reactivity. Macromolecules 11(4): 656662.

12. DR Miller, CW Macosko (1980) Substituent Effects in Property Relations for Stepwise Polyfunctional Polymerization. Macromolecules 13(5): 1063-1069.

13. BE Amborabe, P Fleurat Lessard, JF Chollet, G Roblin (2002) Antifungal Effects of Salicylic Acid and Other Benzoic Acid Derivatives Towards Eutypa lata: Structure-Activity Relationship. Plant Physiology and Biochemistry 40(12): 1051-1060.

14. S Titus, J Hodge (2012) Diagnosis and Treatment of Acne. Am Fam Physician 69(9): 734-740.

15. JF Faig, K Smith, A Moretti, W Yu, KE Uhrich (2016) One-pot Polymerization Synthesis: Incorporation Bioactives into Poly(anhydrideesters). Macromolecular Chemistry and Physics 217(16): 1842-1850. 